\title{
Esclerose Sistêmica e Níveis Séricos Elevados de Organoclorado: Uma Associação Possível?
}

\section{Systemic Sclerosis and Elevated Organochlorine Blood Levels: A Possible Association?}

\author{
Odirlei André Monticielo(1) ${ }^{(1)}$ Penélope Esther Palominos ${ }^{(2)}$, Rafael Mendonça da Silva Chakr ${ }^{(3)}$, \\ Rodrigo Bortoli ${ }^{(4)}$, Ricardo Machado Xavier ${ }^{(5)}$, João Carlos Tavares Brenol ${ }^{(6)}$
}

\section{RESUMO}

A patogênese da esclerose sistêmica (ES) é complexa e pouco conhecida. Há a participação de ativação imune, dano vascular e excessiva produção de matriz extracelular com deposição de colágeno estruturalmente normal. Fatores genéticos e ambientais contribuem para a expressão da doença nos pacientes. Dentre os fatores ambientais, diversos agentes químicos já foram associados à doença. Descrevemos o caso de uma adolescente de 16 anos, com história prévia de hepatite criptogênica e câncer de lábio, que desenvolveu quadro atípico e rapidamente progressivo de esclerose sistêmica. Inicialmente o quadro era compatível com esclerodermia em placas, porém rapidamente evoluiu para uma forma sistêmica com grave acometimento cardiopulmonar e óbito. Durante a investigação de possíveis fatores etiológicos que pudessem estar relacionados, foram encontrados níveis séricos extremamente elevados de oxiclordano, um agrotóxico organoclorado. Uma possível correlação entre a intoxicação por esse agente químico e o surgimento de ES foi aventada.

Palavras-chave: esclerose sistêmica, organoclorado, oxiclordano.

\section{INTRODUÇÃO}

A esclerose sistêmica (ES) é uma doença difusa do tecido conectivo, de natureza auto-imune e caracterizada por fibrose cutânea associada com diversas formas de acometimento visceral. É uma doença rara e estima-se que menos de $10 \%$ dos pacientes com ES desenvolvam a doença antes dos 20 anos de idade ${ }^{(1)}$. Após os 8 anos, as meninas são três vezes mais acometidas do que os meninos, ao passo que, antes dessa

\begin{abstract}
The pathogenesis of systemic sclerosis (SS) is complex and not completely understood. Autoimmune mechanisms, vascular damage, and excessive extracelular matrix with collagen deposition play a significant role. Genetic and environmental factors also are decisive to the disease expression. Among environmental factors many chemical agents have been associated with the development of SS. A 16-year-old white woman, with previous history of cryptogenic hepatitis and cancer of the lips, developed an atypical and rapidly progressive form of SS. Initial sclerodermatous plaques progressed to a systemic form with severe cardiopulmonary involvement and death. Diagnostic workup revealed extremely high blood levels of oxychlordane. A possible association of organochlorine intoxication and SS is proposed.
\end{abstract}

Keywords: systemic sclerosis, organochlorines, oxychlordane.

idade, a incidência é similar em ambos os sexos ${ }^{(2)}$. Na infância, as formas localizadas são as mais comuns e não há relatos de ES induzida por fatores ambientais nessa faixa etária.

Descreve-se aqui o caso de uma adolescente com quadro atípico de esclerodermia em placas que evoluiu para forma sistêmica com grave acometimento cardiopulmonar, sendo descoberta durante a investigação a presença de intoxicação por oxiclordano, o que poderia estar relacionado com a etiopatogênese da doença.

Recebido em 21/6/2007. Aprovado, após revisão, em 14/1 1/2007. Declaramos que os autores Odirlei André Monticielo, Penélope Esther Palominos, Rafael Mendonça da Silva Chakr e Rodrigo Bortoli não apresentam conflitos de interesse. O autor Ricardo Machado Xavier declara honorários de consultoria dos laboratórios Wyeth e Schering-Plough e verba de pesquisa dos laboratórios Abbott e Roché. O autor João Carlos Tavares Brenol declara ser pesquisador e conferencista dos laboratórios Roché, Merk SharpEDome, Zodiac, Novartis, Pfizer, Biogen, EHy Lilly, Wyeth-Bristol e Janssen-Cilag.

Serviço de Reumatologia do Hospital de Clínicas de Porto Alegre, Universidade Federal do Rio Grande do Sul

1. Médico reumatologista, mestrando em Clínica Médica do programa de pós-graduação da Universidade Federal do Rio Grande do Sul (UFRGS).

2. Médica-residente de reumatologia do Hospital de Clínicas de Porto Alegre (HCPA).

3. Médico-residente de reumatologia do HCPA.

4. Médico-residente de reumatologia do HCPA.

5. Professor adjunto de Medicina da UFRGS

6. Professor-associado de Medicina da UFRGS

Endereço para correspondência: Odirlei André Monticielo, Hospital de Clínicas de Porto Alegre, Rua Ramiro Barcelos, 2.350, 6o andar, CEP 90035-903, Porto Alegre, RS, e-mail: omonticielo@ibest.com.br 


\section{DESCRIÇÃO DO CASO}

Paciente do sexo feminimo, branca, 16 anos, procedente da Região Norte do Estado do Rio Grande do Sul, procurou atendimento no ambulatório de Reumatologia do Hospital de Clínicas de Porto Alegre (HCPA) em outubro de 2002, para avaliação de placas esclerodérmicas nas faces flexoras do punho e do ombro direitos com seis meses de evolução. Dois anos antes, havia sido hospitalizada por insuficiência hepática aguda, cuja etiologia não ficou definida, apesar de extensa investigação. Biópsia hepática evidenciou leve necrose em saca-bocado e eosinofilia, e diversos exames foram negativos, tais como anti-HIV, anti-HCV, HBsAg, fator antinuclear (FAN), fator reumatóide (FR), anticorpo antimúsculo liso, anticorpo antimitocondrial, anticorpo antimicrossomal de fígado e rim tipo $l$ e alfa- 1 antripsina sérica. Nova biópsia, realizada no acompanhamento ambulatorial, mostrou hepatite crônica com atividade inflamatória leve e esboço de nódulos regenerativos. No ano anterior à internação, portanto um ano após a hepatite criptogênica, havia sido constatada lesão no lábio inferior, compatível com carcinoma epidermóide verrucoso, tratado com exérese cirúrgica, radioterapia e quimioterapia com cisplatina.

$\mathrm{Na}$ investigação inicial do quadro de esclerodermia em placa, apresentou FAN, FR e anticorpos contra antígenos extraíveis do núcleo (ENA) negativos, ecocardiograma, estudo do trânsito esofágico e provas de função pulmonar, incluindo espirometria, difusão de monóxido de carbono (DCOc) e avaliação dos volumes pulmonares normais. $\mathrm{O}$ raio X de tórax não evidenciou alterações. Biópsias da pele descreviam fibrose da derme profunda e diminuição de fibras colágenas, sem eosinofilia. Naquela ocasião, foram iniciados glicocorticóide intralesional e D-penicilamina, $250 \mathrm{mg} /$ dia.

Em julho de 2004, depois de um longo período sem acompanhamento ambulatorial, foi hospitalizada com progressão importante do quadro cutâneo. Apresentava mãos "em garra”, esclerodactilia, esclerodermia de tronco, antebraços, braços, coxas e dorso dos pés e acometimento facial. Avaliações cardíaca e pulmonar não evidenciaram alterações. Referia fenômeno de Raynaud havia quatro meses. Capilaroscopia periungueal foi inespecífica. Surgiram úlceras no dorso dos pés e em maléolos, cuja biópsia mostrou padrão esclerodermiforme com vasculopatia hialinizante, sem eosinofilia.

A evolução de esclerodermia em placas para ES é muito rara. Diante de um quadro clínico atípico, com história de hepatite criptogênica e neoplasia, foi realizado amplo rastreamento clínico-laboratorial e toxicológico em busca de outras causas de esclerodermia, tais como uso de determinados medicamentos, porfiria cutânea tarda e exposição a agentes tóxicos, lembrando que a paciente era oriunda de zona rural onde há possibilidade de exposição a venenos agrícolas. O diagnóstico histopatológico não sugeria fasceíte eosinofílica. Foram evidenciados níveis séricos muito elevados ( 150 vezes acima do normal) de oxiclordano, um organoclorado comumente usado em lavouras de países fronteiriços com o Brasil. Apesar de a paciente viver em área rural, negou exposição a agentes agrotóxicos. Além disso, os níveis séricos de oxiclordano dos familiares próximos foram normais, embora seu pai apresentasse alterações compatíveis com quadro esclerodermiforme. Apesar da insistência da família e da própria equipe, o pai não procurou atendimento médico.

No seguimento ambulatorial, foi realizado tratamento com ciclofosfamida endovenosa mensal para as manifestações cutâneas, todavia infecções de repetição obrigaram a suspensão. Em junho de 2005, após faltar em algumas consultas, foi internada por infecção respiratória. Exames complementares evidenciaram distúrbio ventilatório obstrutivo (DVO) grave com volume expiratório forçado no primeiro segundo (VEFl) de $700 \mathrm{~mL}$ (29\% do previsto) e alçaponamento aéreo grave. Teste de difusão pulmonar (DCOc) não pôde ser realizado em razão da capacidade vital insuficiente. Ecocardiograma e trânsito esofágico mantinham-se inalterados, e o perfil de auto-anticorpos permaneceu negativo. Tomografia computadorizada de alta resolução (TCAR) mostrou áreas de bronquiectasias e alçaponamento aéreo. Biópsia pulmonar transbrônquica foi sugestiva de bronquiolite obliterante. Em substituição à ciclofosfamida, optou-se pela imunoglobulina humana intravenosa, sendo administradas três doses mensais de $2 \mathrm{mg} / \mathrm{kg}$, com melhora importante das lesões cutâneas, porém sem mudança do quadro pulmonar. Em função da piora respiratória progressiva, foram cogitados transplante autólogo de medula óssea (TMO) e transplante pulmonar, contudo o avançado grau de comprometimento pulmonar inviabilizou tais procedimentos. Evoluiu, então, com dispnéia progressiva, sendo constatada hipertensão arterial pulmonar (HAP) de causa aparentemente não tromboembólica. Posteriormente surgiram hipercapnia grave e hipertensão intracraniana, e o uso de ventilação mecânica não-invasiva com dois níveis de pressão (BiPAP) permitiu melhora parcial da insuficiência ventilatória. Todavia, em 
decorrência de infecção respiratória, a paciente evoluiu para o óbito em junho de 2007.

\section{DISCUSSÃO}

Há três componentes cardinais na patogênese da ES: excesso de colágeno, dano vascular e ativação imunológica ${ }^{(3)}$. A produção e a deposição excessiva de colágeno são expressas principalmente pelo espessamento da pele e pela fibrose pulmonar. O dano vascular caracterizado por proliferação da camada intimal, estreitamento do lúmen e redução do fluxo sanguíneo manifesta-se por fenômeno de Raynaud, crise renal esclerodérmica, úlceras digitais isquêmicas, hipertensão arterial pulmonar e anormalidades vasculares periungueais. Lesão endotelial e disfunção vascular, com aumento dos níveis de endotelina- 1 e diminuição da prostaciclina, costumam ser alterações muito precoces na $\mathrm{ES}^{(4)}$. A ativação imunológica com produção de auto-anticorpos (anticentrômero e antitopoisomerase I ou anti-Scl70) e o processo inflamatório com aumento de citocinas prófibróticas, como fator transformador do crescimento (TGF-beta) e fator de crescimento do tecido conjuntivo (CTGF), compõem também as bases patogênicas da ES até hoje compreendidas ${ }^{(5)}$.

Fatores ambientais e determinantes genéticos participam do desenvolvimento da ES. Estudos mostram que os filhos de pacientes com ES têm um risco pequeno, porém definido, de desenvolver a doença ao longo da vida ${ }^{(1)}$. A influência de determinados haplótipos do HLA e de polimorfismos genéticos em genes específicos reforça a predisposição genética à doença. Infecções como citomegalovirose ${ }^{(6)}$, microquimerismo pós-gestacional ${ }^{(7,8)}$ e diversas substâncias químicas como cloreto de vinil(9), triptofano $^{(10)}$, destilados de petróleo e metissergida ${ }^{(11)}$ poderiam desencadear a cascata de eventos que culminam no surgimento da ES em um indivíduo geneticamente predisposto. Existem relatos sugerindo associação entre fenômeno de Raynaud

\section{REFERÊNCIAS}

1. Medsger Jr. TA: Epidemiology of systemic sclerosis. Clin Dermatol 12(2): 207-16, 1994.

2. Martini G, Foeldvari I, Russo R, et al.: Systemic sclerosis in childhood: clinical and immunologic features of 153 patients in an international database. Arthritis Rheum 54(12): 3971-8, 2006.

3. Clement PJ: Systemic Sclerosis. Baltimore: Williams \& Wilkins, 1996.

4. Schachna L, Wigley FM: Targeting mediators of vascular injury in scleroderma. Curr Opin Rheumatol 14(6): 686-93, 2002. e a administração de certos agentes quimioterápicos, como bleomicina, vincristina e cisplatina ${ }^{(12)}$. Recentemente, foram relatados casos de esclerodermia em pacientes oncológicos tratados com taxanos ${ }^{(13)}$. Em 2007, uma metanálise de estudos de caso-controle constatou risco aumentado de ES relacionado à exposição ocupacional a solventes ${ }^{(14)}$. Até o momento, a relação entre intoxicação por organoclorado e ES não foi estabelecida.

Organoclorados são compostos lipofílicos com meia-vida prolongada, utilizados como pesticidas para o controle de pragas domiciliares e agrícolas e no controle de cupins. Após exposição prolongada, mesmo com doses relativamente baixas, podem acumular-se no tecido adiposo e causar toxicidade ${ }^{(15)}$. Há relatos na literatura de que o oxiclordano possa estar associado a risco aumentado de algumas patologias como câncer de próstata ${ }^{(16)}$ e linfoma não-Hodgkin ${ }^{(17)}$. Um estudo realizado com roedores portadores de lúpus eritematoso sistêmico induzido laboratorialmente mostrou taxa de progressão mais rápida para nefrite lúpica nos animais expostos a organoclorados, sendo questionado se as propriedades estrogênicas de alguns organoclorados poderiam contribuir para a progressão da auto-imunidade ${ }^{(18)}$.

Deve-se ressaltar, ainda, a já estabelecida associação entre cisplatina e manifestações esclerodérmicas ${ }^{(19)}$. Cronologicamente é viável a associação entre o uso de cisplatina e o desenvolvimento de ES no caso em questão, porém não se pode afastar um sinergismo entre ela e o oxiclordano, tendo em vista a forma atípica e rapidamente progressiva de apresentação da doença, diferentemente dos quadros descritos na literatura. Além disso, por seu potencial hepatotóxico e oncogênico, a intoxicação por oxciclordano poderia explicar a hepatite criptogênica e o surgimento do carcinoma epidermóide de lábio. Contudo, estudos que possam determinar melhor nexo de causalidade são necessários para estabelecer o real papel desse agente no desenvolvimento da doença.

5. Ihn H: Pathogenesis of fibrosis: role of TGF-beta and CTGF. Curr Opin Rheumatol 14(6): 681-5, 2002.

6. Lunardi C, Bason C, Navone R, et al.: Systemic sclerosis immunoglobulin $\mathrm{G}$ autoantibodies bind the human cytomegalovirus late protein UL94 and induce apoptosis in human endothelial cells. Nat Med 6(10): 1183-6, 2000.

7. Jimenez SA, Artlett CM: Microchimerism and systemic sclerosis. Curr Opin Rheumatol 17(1): 86-90, 2005.

8. Charles CP, Clements, Furst DE: Systemic sclerosis: hypothesisdriven treatment strategies. Lancet 367(9523): 1683-91, 2006. 
9. Veltman G, Lange CE, Jühe S, Stein G, Bachner U: Clinical manifestations and course of vinyl chloride disease. Ann N Y Acad Sci 246: 6-17, 1975.

10. Silver RM, Heyes MP, Maize JC, Quearry B, Vionnet-Fuasset M, Sternberg EM: Scleroderma, fasciitis, and eosinophilia associated with the ingestion of tryptophan. $\mathrm{N}$ Engl J Med 322(13): 874-81, 1990.

11. Kluger N, Girard C, Bessis D, Guillot B: Methysergide-induced scleroderma-like changes of the legs. Br J Dermatol 153(1): 224-5, 2005.

12. Adoue D, Arlet P, Vilain C, Le Tallec Y, Bonafé JL, de Lafontan B: Raynaud's phenomenon after chemotherapy. Apropos of 3 cases. Ann Dermatol Venereol 112(2): 151-5, 1985.

13. Itoh M, Yanaba K, Kobayashi T, Nakagawa H: Taxane-induced scleroderma. Br J Dermatol 156(2): 363-7, 2007.

14. Kettaneh A, Al Moufti O, Tiev KP, et al.: Occupational exposure to solvents and gender-related risk of systemic sclerosis: a metaanalysis of case-control studies. J Rheumatol 34(1): 97-103, 2007.

15. Dich J, Al Moufti O, Tiev KP, et al.: Pesticides and cancer. Cancer Causes Control 8(3): 420-43, 1997.

16. Ritchie JM, Vial SL, Fuortes LJ, Guo H, Reedy VE, Smith EM: Organochlorines and risk of prostate cancer. J Occup Environ Med 45(7): 692-702, 2003.

17. Quintana PJ, Delfino RJ, Korrick S, et al.: Adipose tissue levels of organochlorine pesticides and polychlorinated biphenyls and risk of non-Hodgkin's lymphoma. Environ Health Perspect 112(8): 854-61, 2004.

18. Sobel ES, Gianini J, Butfiloski EJ, Croker BP, Schiffenbauer J, Roberts SM: Acceleration of autoimmunity by organochlorine pesticides in (NZB x NZW)Fl mice. Environ Health Perspect 113(3): 323-8, 2005.

19. Emir S, Kutluk T, Topaloğlu R, Bakkaloğlu A, Büyükpamukçu M. Scleroderma in a child after chemotherapy for cancer. Clin Exp Rheumatol 19(2): 221-3, 2001. 